\title{
Monocular 3-D Tracking of the Golf Swing*
}

\author{
Raquel Urtasun ${ }^{\dagger} \quad$ David Fleet $^{\ddagger} \quad$ Pascal Fua $^{\dagger}$ \\ $\dagger$ Computer Vision Laboratory \\ Swiss Federal Institute of Technology (EPFL) \\ 1015 Lausanne, Switzerland \\ Email: \{raquel.urtasun, pascal.fua\}@epfl.ch \\ $\ddagger$ Department of Computer Science \\ University of Toronto \\ M5S 3H5, Canada \\ Email: fleet@cs.toronto.edu
}

Technical Report No: IC/2004/90

\begin{abstract}
We propose an approach to incorporating dynamic models into the human body tracking process that yields full 3-D reconstructions from monocular sequences.

We formulate the tracking problem is terms of minimizing a differentiable criterion whose differential structure is rich enough for successful optimization using a single-hypothesis hill-climbing approach as opposed to a multi-hypotheses probabilistic one. In other words, we avoid the computational complexity of multi-hypotheses algorithms while obtaining excellent results under challenging conditions.

To demonstrate this, we focus on monocular tracking of a golf swing from ordinary videos. It involves both dealing with potentially very different swing styles, recovering arm motions that are perpendicular to the camera plane and handling strong self-occlusions.
\end{abstract}

\section{Introduction}

In spite of having received considerable attention in recent years, monocular tracking of human motion remains a difficult problem, especially in the presence of self-occlusions and movements perpendicular to the image plane.

Most current approaches rely on multi-hypotheses optimization techniques $[11,9,8,6,20]$ to resolve the inherent ambiguities of this problem and to escape the local-minima that are usually involved. They have been shown to be effective but require ever increasing computational burdens as the number of degrees of freedom in the model increases.

In earlier work [22, 23], we have advocated the use of temporal motion models based on Principal Component Analysis (PCA) and inspired by those proposed in $[17,19]$ to formulate the tracking problem as one of minimizing

\footnotetext{
*This work was supported in part by the Swiss Federal Office for Education and Science.
} 

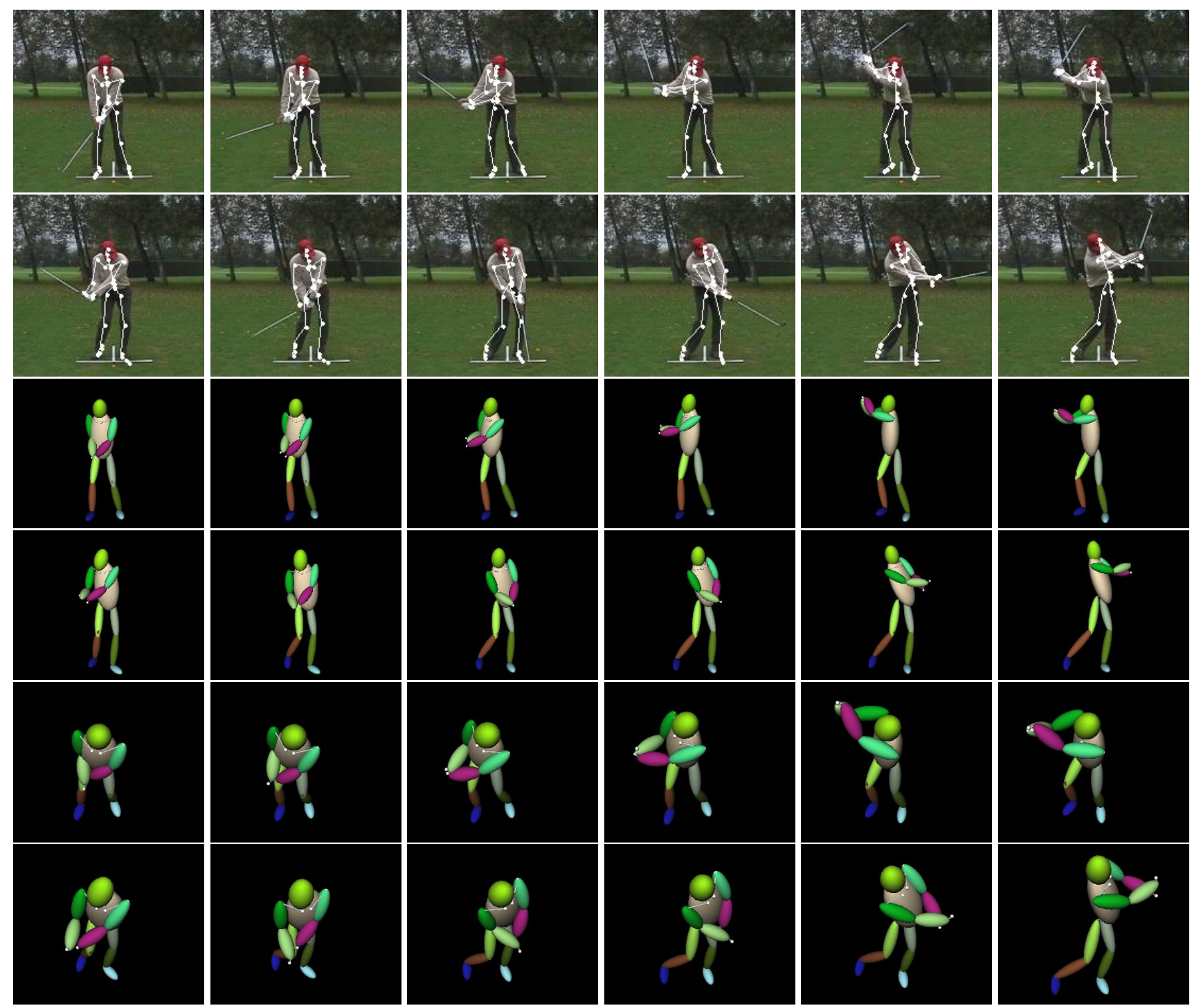

Figure 1: Tracking a driving swing in a 45 frames sequence. First two rows: The skeleton of the recovered 3-D model is projected into a representative subset of images. Middle two rows: Volumetric primitives of the recovered 3-D model projected into the same views. Bottom two rows: Volumetric primitives of the 3-D model as seen from above.

differentiable objective functions when using stereo data. The differential structure of these objective functions is rich enough to take advantage of standard hill-climbing optimization methods, whose computational requirements are much smaller than those of multiple-hypotheses ones and can nevertheless yield very good results.

Here, as shown in Figs. 1, 10, and 11, we extend this approach to monocular tracking, and demonstrate its ability to track such a complex fully 3-D motion as a golf swing. Unlike some recent approaches to incorporating dynamic models in 2-D [2], we recover full 3-D from a single fixed camera. As shown in the bottom rows of Fig. 1, this is important for golf because, at the top of the swing, the arm motion perpendicular to the camera plane is both large and very significant.

Of course, it could be argued that by using a strong motion model, we constrain the problem to the point where it becomes almost trivial. We will show that this is most definitely not the case and that our model still has sufficient flexibility not only to model very different golf swings, such as those of Figs. 1 and 11, but also to produce totally 
meaningless results if the image data is not properly exploited. In other words, our implementation embodies a happy middle ground between an over-constrained model that is too inflexible and one that is so loose that it makes the optimization very difficult. In our experience [23] with walking, running, and jumping, we found these activities to be amenable to the kind of modeling we use here. We therefore believe our approach to be applicable not only to golf but also to many other motions, in particular athletic ones, that involve predictable movements.

In the remainder of this paper we first discuss related approaches and introduce our deterministic motion model. We then show how we use it to incorporate the kind of image information that can actually be extracted from video sequences acquired on golf courses under uncontrolled circumstances. Finally, we discuss our results in more detail and propose avenues for future research.

\section{Related Work}

Modeling the human body and its motion is of enormous interest in the Computer Vision community, as attested by recent, exhaustive, and already dated surveys $[15,14]$. However, existing techniques remain fairly brittle for many reasons: Humans have a complex articulated geometry overlaid with deformable tissues, skin and loose clothing. They move constantly, and their motion is often rapid, complex and self-occluding. Furthermore, the 3-D body pose is only partially recoverable from its projection in one single image. Reliable 3-D motion analysis therefore requires reliable tracking across frames, which is difficult because of the poor quality of image-data and frequent occlusions. Recent approaches to handling these problems can roughly be classified into those that

- Detect: This implies recognizing postures from a single image by matching it against a database and has become increasingly popular recently $[1,10,16,21]$ but requires very large sets of examples to be effective.

- Track: This involves predicting the pose in a frame given observation of the previous one. This can easily fail if errors start accumulating in the prediction, causing the estimation process to diverge. This is usually mitigated by introducing sophisticated statistical techniques for a more effective search $[11,9,8,6,20]$ or by using strong dynamic motion models as priors [17, 19,2].

Neither technique is proven as superior, and both are actively investigated. However, the tracking approach is the most natural one to use when a person is known a priori to be performing a given activity, such as walking, running, or jumping. Introducing a motion model becomes an effective means to constrain the search and increase robustness. Furthermore, instead of a separate pose in each frame, the output are the parameters of the motion model, which allow for further analysis and are therefore potentially more useful.

Models that represent motion vectors as linear sums of principal components are of particular interest to us and have been used effectively to produce realistic computer animations [3, 5, 4]. The PCA components are computed by capturing as many people as possible performing a specific activity, for example by means of an optical motion capture system, representing each motion as a temporally quantized vector of joint angles, and performing a Principal Component Analysis on the resulting set of vectors.

This representation has already been successfully used in our community [17, 19], but almost always in a statistical context and without exploiting the fact that this parameterization allows the formulation of the tracking problem as one of minimizing differentiable objective functions, which allows for a lower computational complexity.

\section{Motion Model}

We represent the body of the golfer as a set of volumetric primitives attached to an articulated 3-D skeleton, as shown in the bottom rows of Fig. 1. Its pose is given by the position and orientation of its root node and a set of 

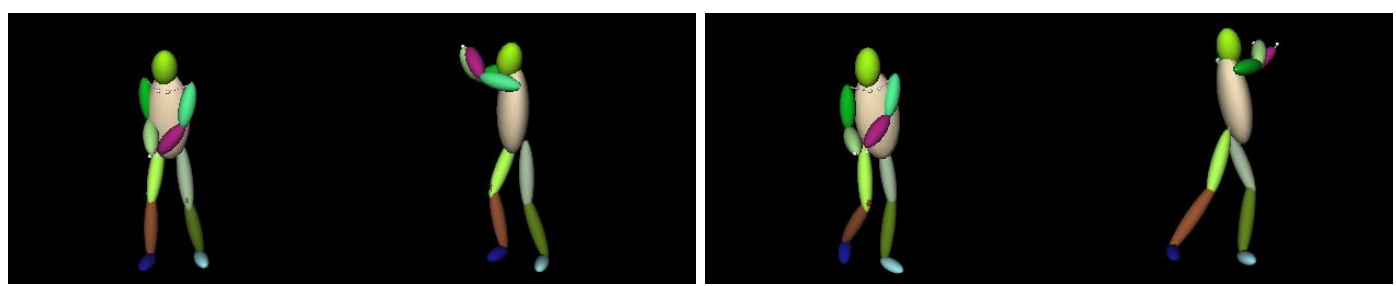

Figure 2: Key postures. From top left: Beginning of upswing, end of upswing, ball hit, and end of downswing.

joint angles. To build a motion model, we used the ten golf swing motions of the CMU database [7]. We identified the 4 key postures depicted by Fig. 2 in each motion and time warped the swings so that the key postures are all reached at the same time. We then sampled them at regular time intervals using quaternion spherical interpolation so that each swing can be treated as $N=200$ samples of a motion starting at normalized time 0 and ending at normalized time 1.

A swing is then represented by an angular motion vector $\Psi$ of size $N * N$ Dofs, where $N$ Dof $s=72$ is the number of angular degrees of freedom in the body model. $\Psi$ is a line vector of the form:

$$
\Psi=\left[\psi_{\mu_{1}}, \cdots, \psi_{\mu_{N}}\right]
$$

where the $\psi_{\mu_{i}}$ are line vectors representing the joint angles at normalized time $\mu_{i}$. The posture at a given time $0 \leq \mu_{t}<1$ is estimated by interpolating the values of the $\psi_{\mu_{i}}$ corresponding to postures immediately before and after $\mu_{t}$.

Assuming our set to be representative, a motion vector $\Phi$ can be approximated as a weighted sum of the mean motion $\Theta_{0}$ and the eigenvectors $\Theta_{i}$ of the covariance matrix:

$$
\Psi \approx \Theta_{0}+\sum_{i=1}^{m} \alpha_{i} \Theta_{i}
$$

where the $\alpha_{i}$ are scalar coefficients that characterize the motion, $m \leq M$ controls the percentage of the database that can be represented in this manner and $M=10$ is the number of examples. For the small database we use, we have found $m=4$ to be an appropriate value to use. As will be discussed in Section 5.2, the database is clearly too small and we plan to augment it. However, based on previous experience with walking, running and jumping [23], we do not expect the required value of $m$ to grow dramatically for the specific purpose of modeling golf swings, or more generally constrained athletic motions.

As will be discussed in Section 4, our tracking is defined as the least-squares minimization [18] of an objective function $F$ with respect to the motion model parameters $\alpha_{i}, \mu_{t}$ and the global motion $G_{t}$ of the skeleton's root node, defined at the level of the sacroiliac, that is not included in the motion model. This involves computing the Jacobian of $F$. Assuming that $\frac{\partial F}{\partial \theta_{j}}$ is differentiable, i.e. that the derivatives of $F$ with respect to the individual joint angles $\theta_{j}$ exist, this can be readily computed as follows:

$$
\begin{gathered}
\frac{\partial F}{\partial \alpha_{i}}=\sum_{j=1}^{n d o f} \frac{\partial \theta_{j}}{\partial \alpha_{i}} \cdot \frac{\partial F}{\partial \theta_{j}} \\
\frac{\partial F}{\partial \mu_{t}}=\sum_{j=1}^{n d o f} \frac{\partial \theta_{j}}{\partial \mu_{t}} \cdot \frac{\partial F}{\partial \theta_{j}},
\end{gathered}
$$

where $\frac{\partial \theta_{j}}{\partial \alpha_{i}}$ and $\frac{\partial \theta_{j}}{\partial \mu_{t}}$ are computed as in [22]. 


\section{Least Squares Framework}

Given that we operate outdoors in an uncontrolled environment and want to track golfers who are wearing their normal clothes, we cannot rely on any one image clue to give us all the information we need. Instead, we take advantage of several sources of information, none of which is perfect, but that together have proved sufficient for our purposes.

More specifically, we sequentially fit our motion model over sliding groups of $f$ frames. For such a set of $f$ frames, we take the state vector $\mathbf{S}$, to be

$$
\mathbf{S}=\left[\alpha_{1}, \ldots, \alpha_{M}, \mu_{1}, \ldots, \mu_{f}, G_{1}, \ldots, G_{f}\right],
$$

where the $\alpha_{i}$ are the PCA weights of Section 3 common to the set of $f$ frames, the $\mu_{t}$ are the normalized time associated to each frame, and the $G_{t}$ represent the corresponding absolute position and orientation of the root node that vary in every frame.

We use the image data to write $n_{\mathrm{obs}}$ observation equations of the form

$$
\operatorname{Obs}\left(\mathbf{x}_{i}, \mathbf{S}\right)=\epsilon_{i}, 1 \leq i \leq n_{\mathrm{obs}},
$$

where $\mathbf{x}_{i}$ is a data point, Obs a differentiable function whose value is zero for the correct value of $\mathbf{S}$ and completely noise free data, and $\epsilon_{i}$ is treated as an independently distributed Gaussian error term. We then minimize $v^{T} P v$, where $v=\left[\epsilon_{1}, \ldots, \epsilon_{n_{\mathrm{obs}}}\right]$ is the vector of residuals and $P$ is a diagonal weight matrix associated with the observations. Our system must be able to deal with observations coming from different sources that may not be commensurate with each other. We therefore associate to each data point $\mathbf{x}_{i}$ an observation type type $e_{i}$ and to each type a weight $w^{\text {type }}$ corresponding to the importance of the observation type.

Because the image data is noisy, we add a regularization term $E_{D}$ that forces the motion to remain smooth. The total energy that we minimize therefore becomes:

$$
\begin{aligned}
E_{T}= & \sum_{i=1}^{\text {nobs }} w^{\text {type }_{i}}\left\|\operatorname{Obs}^{\text {type }}\left(\mathbf{x}_{i}, \mathbf{S}\right)\right\|^{2}+E_{D} \\
E_{D}= & w_{G}\left(G_{t}-\hat{G}_{t}\right)+w_{\mu}\left(\mu_{t}-\hat{\mu}_{t}\right) \\
& +w_{\alpha} \sum_{i=1}^{m}\left(\alpha_{i}-\hat{\alpha}_{i}\right)
\end{aligned}
$$

where $O b s^{t y p e}$ is the function that corresponds to a particular observation type, $\hat{G}^{t}$ and $\hat{\mu}_{t}$ are predicted values for the position and orientation of the root node and the predicted normalized time, and $w_{G}, w_{\mu}$ and $w_{\alpha}$ are scalar weights. We take $\hat{G}^{t}$ to be $G^{t-1}+\Delta G^{t-1}$ and $\hat{\mu}_{t}$ to be $\mu_{t-1}+\Delta \mu_{t-1}$, where $\Delta G^{t-1}, \Delta \mu_{t-1}$ are the speeds observed in the previous set of frames.

We now turn to the description of the $O b s^{\text {type }}$ functions for the data types we use and conclude the section by describing their complementarity.

\subsection{Foreground and Background}

Given an image of the background without the golfer, we can extract rough binary masks of the foreground such as those of Fig. 3. Note that because the background is not truly static, they cannot be expected to be of very high quality. Nevertheless, they can be exploited as follows. We sample them and for each sample $\mathbf{x}$ we define a Background/Foreground function $\mathrm{Obs}^{f g / b g}\left(\mathbf{x}_{i}, \mathbf{S}\right)$ that is 0 if the line of sight defined by $\mathbf{x}$ intersects the model and is equal to the distance of the model to the line of sight otherwise. In other words, $O b s^{f g / b g}$ is a differentiable 


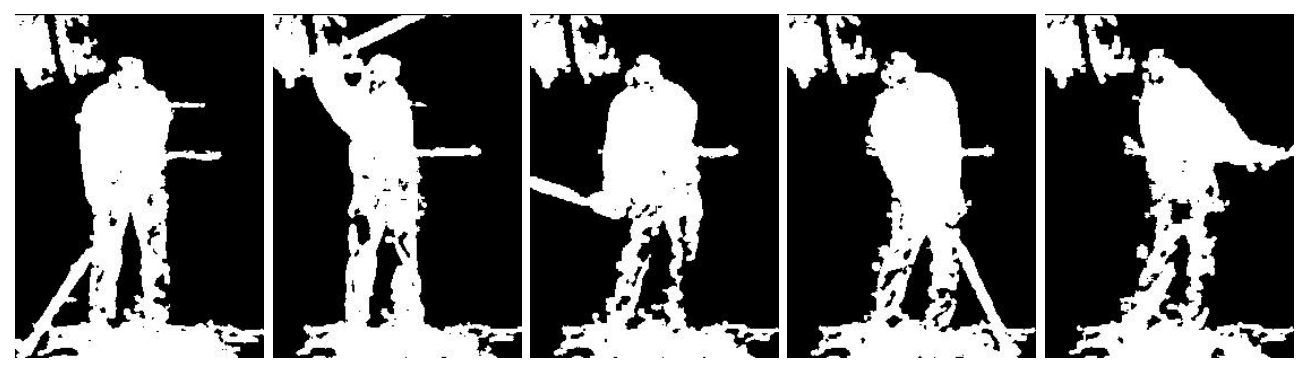

Figure 3: Poor quality foreground binary mask extracted from the images of Fig. 10.
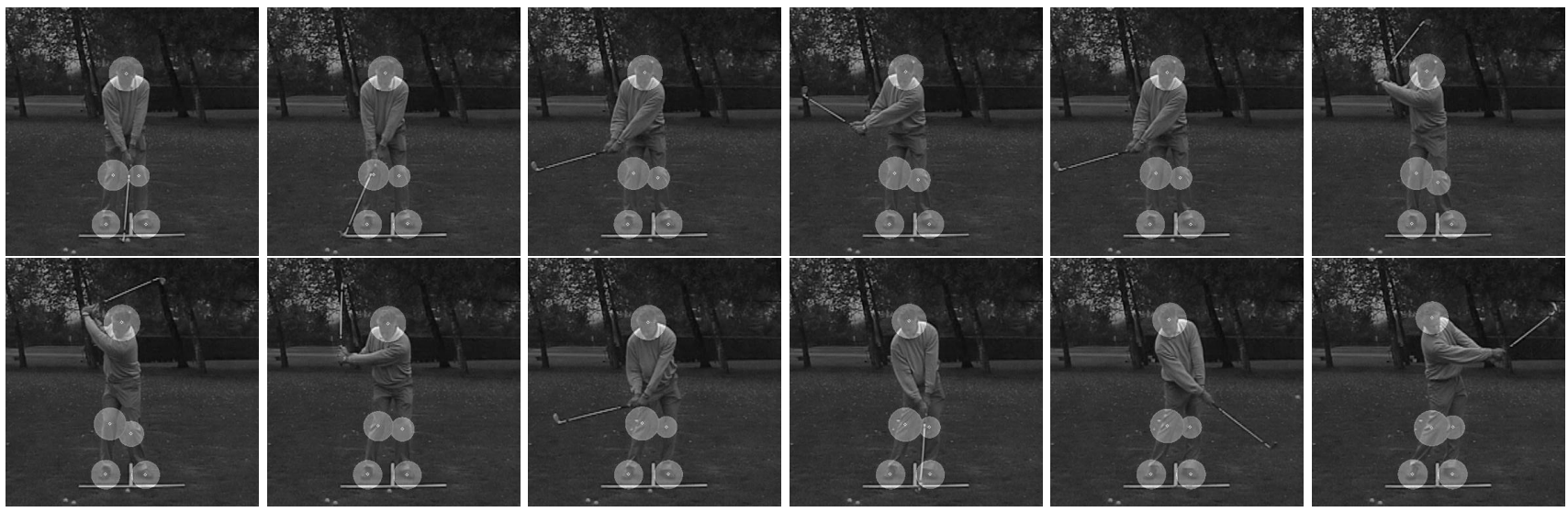

Figure 4: 2-D tracking of the ankles, knees, and vc2, using the WSL appearance-based tracker.

function that introduces a penalty for each point in the foreground binary mask that does not backproject to the model. That penalty increases with the 3-D distance of the model to the corresponding line of sight.

Minimizing $O b s^{f g / b g}$ in the least squares sense tends to maximize the overlap between the model's projection and the foreground binary mask. This prevents the pose estimates from drifting, potentially resulting in the model eventually projecting at the wrong place and tracking failure.

\subsection{Projection Constraints}

To further constrain the location of six key joints - knees, ankles and wrists — and the head, we track their approximate image projections across the sequence.

As shown in Fig. 4, for the ankles, knees and head, we use the WLS tracker [12] to take advantage of the slow dynamics of changes in image patches. WSL is a robust, motion-based 2-D tracker that maintains an online adaptive appearance model. The model adapts to slowly changing image appearance with a natural measure of the temporal stability of the underlying image structure. By identifying stable properties of appearance the tracker can weight them more heavily for motion estimation, while less stable properties can be proportionately downweighted.

For the wrists, because the hand tends to rotate during the motion, we have found it more effective to use a club tracking algorithm [13] that takes advantage of the information provided by the whole shaft. It is depicted by Fig. 5 and does not require any manual initialization. It is also very robust to mis-detections and false alarms and has been validated on many sequences. Hypotheses on the position are first generated by detecting pairs of close parallel segments in the frames, and then robustly fitting a 2D motion model over several frames simultaneously. From the recovered club motion, we can infer the 2-D hand trajectories of Fig. 6. 

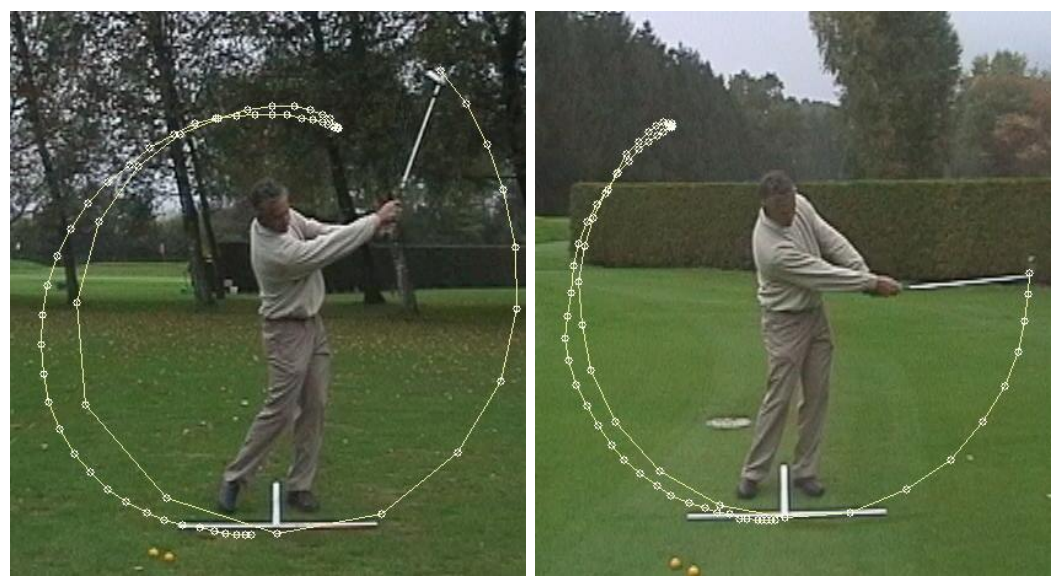

Figure 5: Detected club trajectories for the driving swing of Fig. 1 and the approach swing of Fig. 10. Note that one trajectory is much more extended than the other.

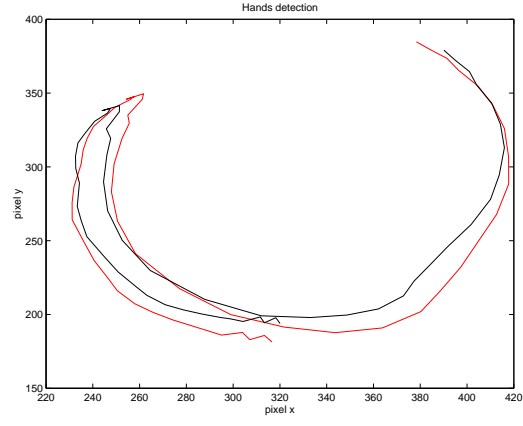

(a)

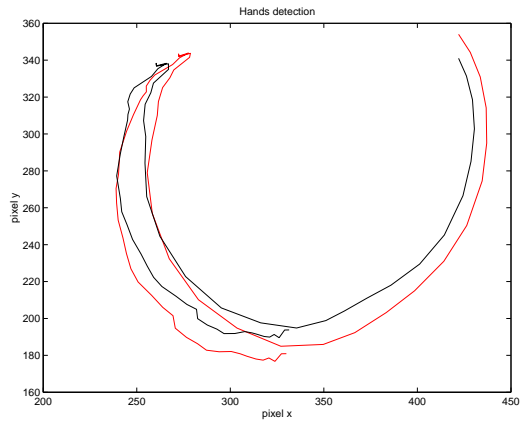

(b)

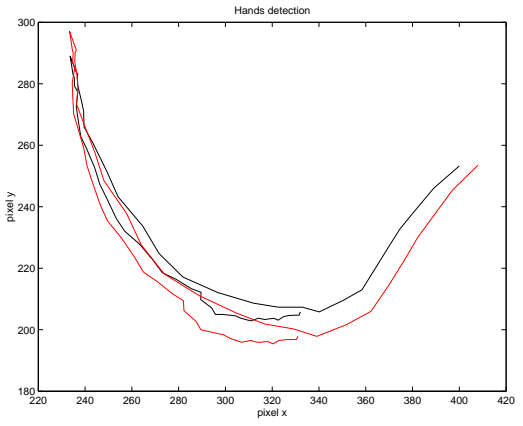

(c)

Figure 6: Detected hand trajectories for drive and approach swings. The left and right hands are represented in black and red respectively. Trajectories in the sequence of (a) Fig. 1. (b) Fig. 10. (c) Fig. 11. Note the difference between the last one that corresponds to an approach swing and the first two that correspond to driving swings.

For joint $j$, we therefore obtain approximate $2-\mathrm{D}$ positions $\mathbf{x}_{t}{ }^{\mathbf{j}}$ in each frame. Given that the joint's $3-\mathrm{D}$ position and therefore its projection are a function of $S$, we simply take the corresponding joint projection function $\mathrm{Obs}^{\text {joint }}\left(\mathbf{x}^{\mathbf{j}}, \mathbf{S}\right)$ to be the Euclidean distance between the joint projection and its estimated 2-D location.

\subsection{Point Correspondences}

We use 2-D point correspondences in pairs of consecutive images as an additional source of information: We project the 3-D model into the first image of the pair, sample the projection, and establish correspondences for those samples in the second one using a simple correlation-based algorithm. Given a couple $\mathbf{x}_{i}=\left(p_{i}^{1}, p_{i}^{2}\right)$ of corresponding points found in this manner, we define a correspondence function $\operatorname{Obs}^{\operatorname{corr}}\left(\mathbf{x}_{i}, \mathbf{S}\right)$ as follows: We backproject $p_{i}^{1}$ to the 3-D model surface and reproject it to the second image. We then take $O b s^{\operatorname{corr}}\left(\mathbf{x}_{i}, \mathbf{S}\right)$ to be the Euclidean distance in the image plane between this reprojection and $p_{i}^{2}$.

\subsection{Complementarity of the Objective Function Terms}

As discussed above the foreground/background observations of Section 4.1 stop the estimates from drifting by guaranteeing that the model keeps on projecting roughly at the right place. The projection observations of Sec- 

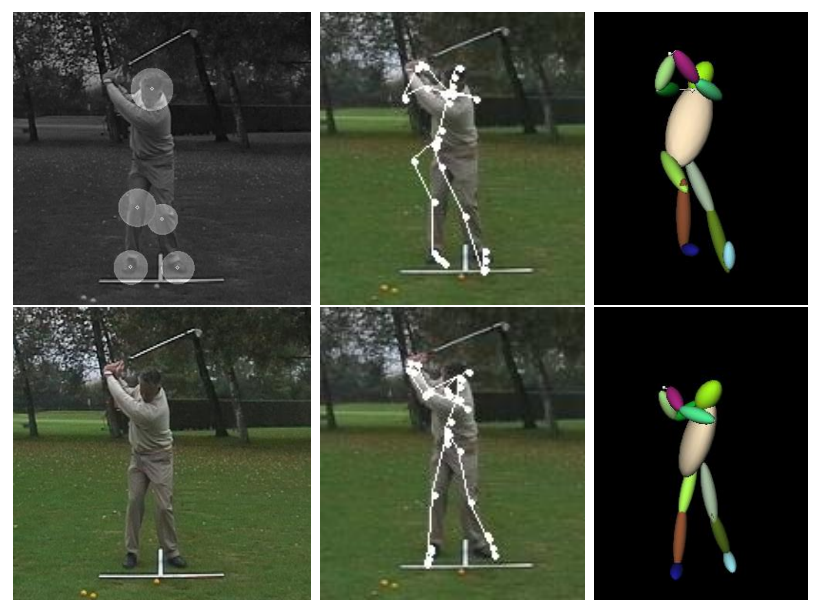

Figure 7: Tracking using only joint constraints vs using the complete objective function. (a) Using only joint constraints the problem is under-constrained and a multiple set of solutions are possible. (b) The set of solutions is reduced using correspondences.
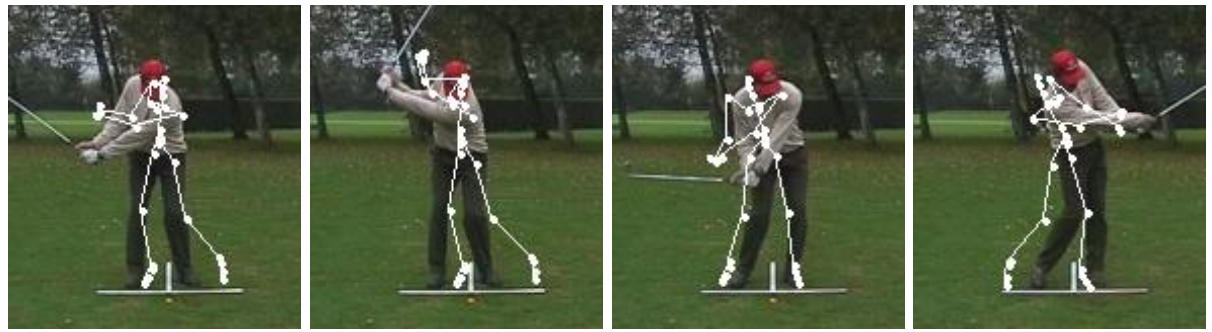

Figure 8: Mean motion used for initialization.

tion 4.2 more precisely constrain the projections of the ankles, knees, wrists and head.

However, as shown in the top row of Fig. 7, these two sets of constraints are not sufficient by themselves. The correspondences of Section 4.3 are required to fully constrain the motion of both the lower and upper body. Of course, the correspondences by themselves would not be enough either: They are too noisy to be used alone because the golfer is wearing untextured clothing and the wrinkles produce correspondence motion that does not necessarily follow the golfer's true motion.

The example of Fig. 7 is significant because it shows that the model has sufficient flexibility to do the wrong thing given insufficient image data. In other words, even though we use a motion model, the problem is not so constrained that we are guaranteed to get valid postures or motions without using the images correctly.

\section{Tracking}

In this section, we first discuss the initialization of our tracking procedures, which only requires a minimal amount of manual intervention. We then present our results in more detail. 

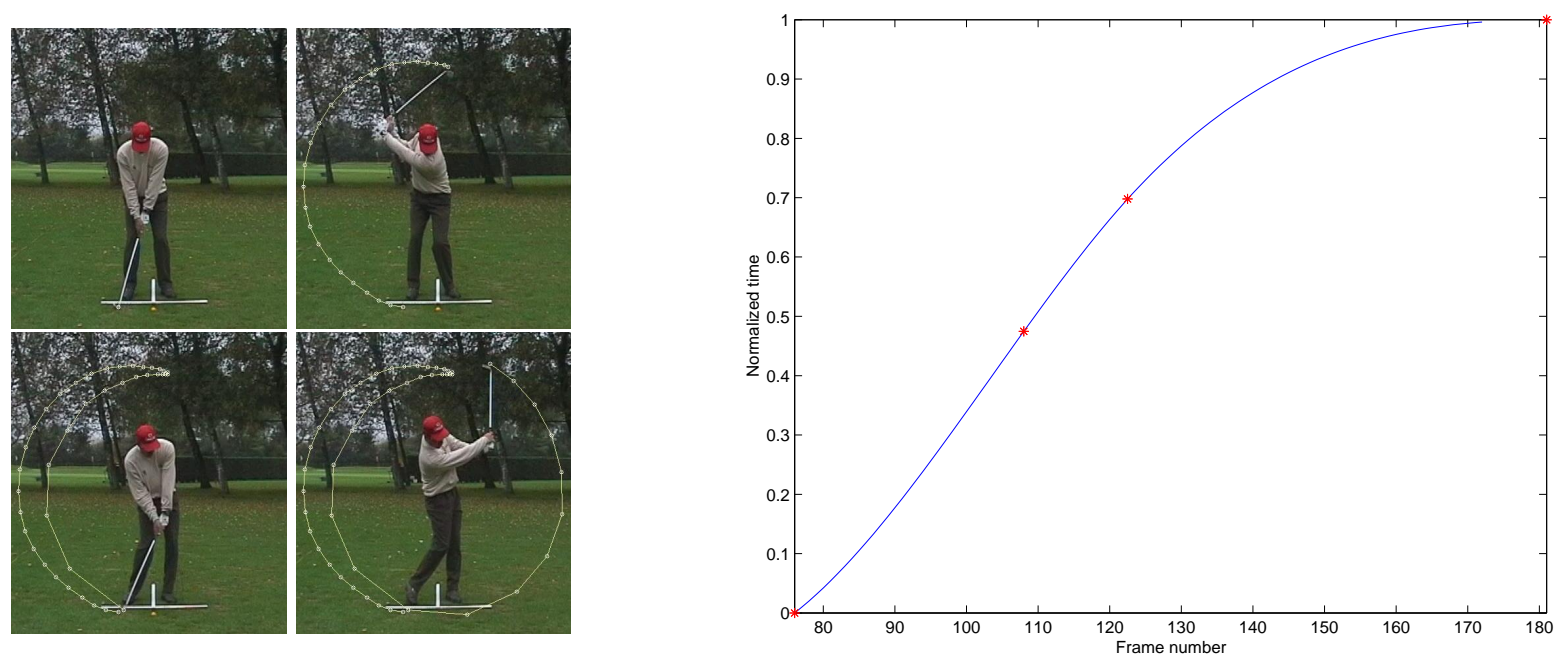

(a)

(b)

Figure 9: Assigning normalized times to the frames of Fig. 1. (a) We use the automatically detected club positions to identify the key postures of Fig. 2. (b) The corresponding normalized times are denoted by red dots. Spline interpolation is then used to initialize the $\mu_{t}$ parameter for all other frames in the sequence.

\subsection{Initialization}

For each sequence, we first run the golf club tracker [13] discussed in Section 4.2. As shown in Fig. 9(a), the detected club positions let us initialize the $\mu_{t}$ parameters by telling us in which four frames the key postures of Fig 2 can be observed. As discussed in Section 3, the corresponding normalized times were defined upon creating the database. We can therefore assign a normalized time to all other frames in the sequence by spline interpolation, as shown in Fig. 9(b). As not everybody performs the motion at the same speed, this time is only a guess and will be refined during the actual optimization.

We then roughly position the root node of the body so that it projects approximately at the right place in the first frame and specify in that first frame the locations of the five joints to be tracked by WSL [12]. Note that all of this only requires a few mouse clicks and could easily be automated using posture detection techniques, given the fact that the position at the beginning of a swing is completely stereotyped.

We can now start the tracking algorithm by setting all the PCA weights to zero and minimizing in a fully automated fashion the criterion of Eq. 7 three frames at a time. Note that, as shown in Figure 8, the mean motion and the interpolated values of $\mu_{t}$, do not yield an initially correct motion. The style of the swing is different, the speed of the motion varies between different golfers, and optimization of the criterion of Eq. 7 is truly required.

\subsection{Results}

Figs. 1 and 10 depict complete driving swings performed by two different subjects whose motions were not recorded in the CMU database [7]. In both cases, we show projections of the recovered 3D model in a representative subset of the images. In Fig. 1, we also display the recovered 3D model, first projected in the original view and then as seen from above. Note the quality of the tracking in spite of the facts that the golfers are wearing relatively untextured clothing, their sizes are unknown and the cameras uncalibrated. To perform our computation, we used rough estimates of both the subjects size and the cameras focal length. In practice, this information could be made available to the system, thereby simplifying its task.

Fig 11 depicts a much shorter approach swing, where the club does not go as high as in a driving swing, as evidenced by the hand trajectories of Fig. 6. This is challenging for our system because the CMU database only contains driving swings. Our model nevertheless has sufficient flexibility to generalize to this new motion. Note, 

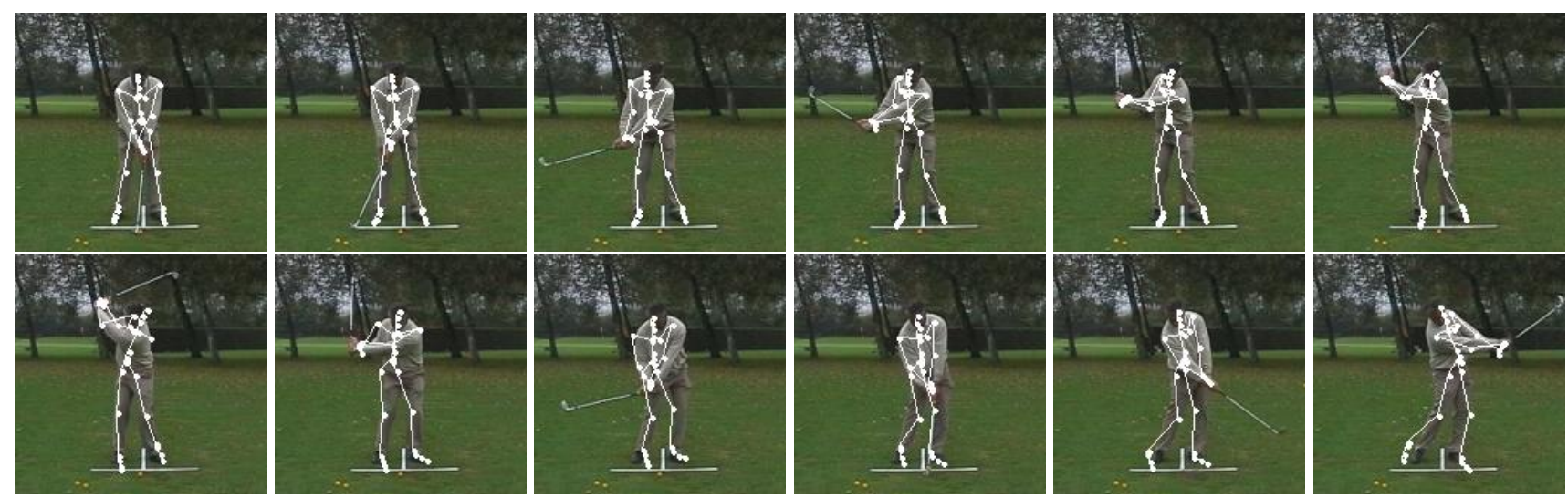

Figure 10: Tracking another driving swing. The skeleton of the recovered 3-D model is projected onto the images.
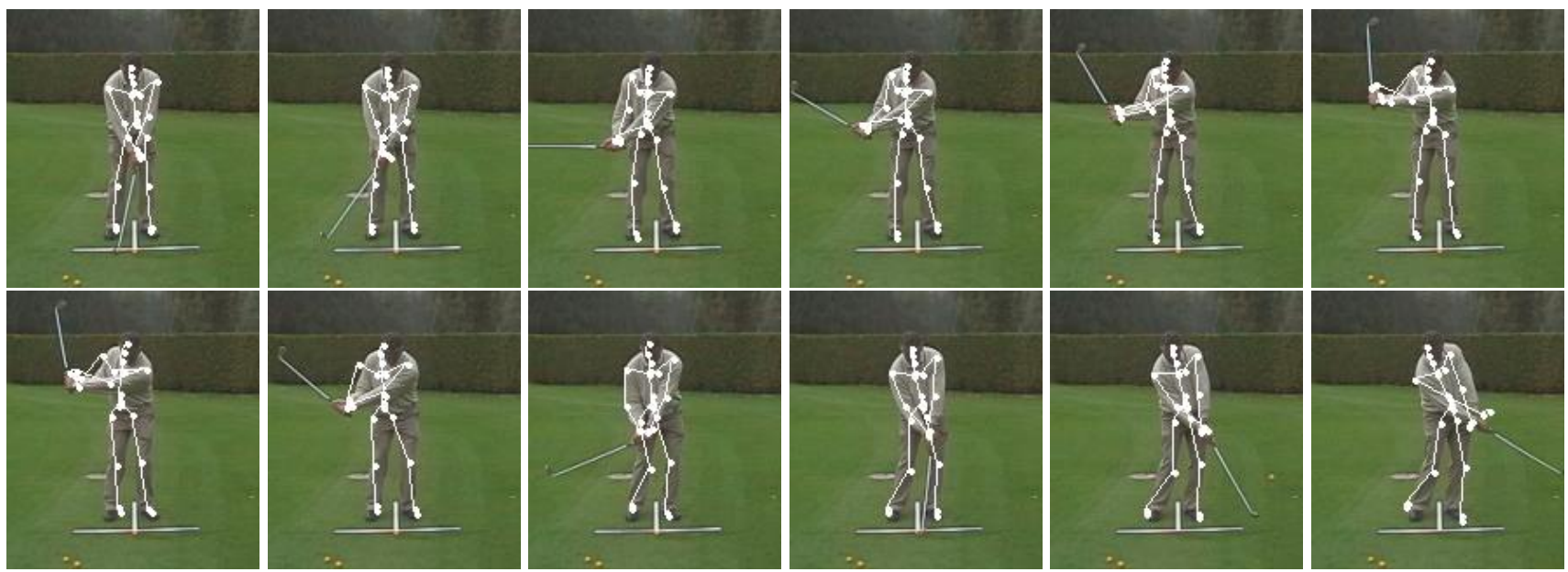

Figure 11: Tracking an approach swing during which the club goes much less high than in a driving swing. The skeleton of the recovered 3-D model is projected onto the images.

however, that the right leg bends slightly too much at the end of the motion, which is a reflection of the small size of the database and of the fact that all the exemplars in it bend their legs in this particular fashion. The obvious cure for this problem is to use a much more complete database, which we intend to build in the very near future using a VICON ${ }^{t m}$ optical motion capture system we have access to.

\section{Conclusion}

We have presented an approach to incorporating strong motion models that yields full 3-D reconstructions from monocular sequences using a single-hypothesis hill-climbing approach, which results in much lower computational complexity than current multi-hypotheses techniques.

We have demonstrated it for monocular tracking of a golf swing from ordinary videos, which involves dealing with potentially very different swing styles, recovering arm motions that are perpendicular to the camera plane, and handling strong self-occlusions. The major limitation of the current implementation stems from the small size 
of the motion database we used, which we will remedy in the coming months.

We have obviously placed ourselves in a relatively constrained context, which is nevertheless far from simple and makes perfect sense in terms of potential industrial applications. Furthermore, we believe there is also ample scope for broadening this approach given a "library" of models such as the ones we have used here or those we developed in our earlier walking, running and jumping work [22, 23]: In a broader context, with specific motion models, we have traded the complexity of tracking for the complexity of knowing which model to apply. This might mean keeping several models active at any one time and selecting the one that fits best. This brings us back to multiple hypotheses tracking, but the multiple hypotheses are over models and not states. This might be much more effective than what many particle filters do because it ensures that the multiple hypotheses are sufficiently different to be worth exploring. This is an avenue of research we intend to pursue in future work.

\section{References}

[1] A. Agarwal and B. Triggs. 3d human pose from silhouettes by relevance vector regression. In Conference on Computer Vision and Pattern Recognition, 2004.

[2] A. Agarwal and B. Triggs. Tracking articulated motion with piecewise learned dynamical models. In European Conference on Computer Vision, pages III 54-65, Prague, May 2004.

[3] M. Alexa and W. Mueller. Representing animations by principal components. In Eurographics, volume 19, 2000.

[4] V. Blanz, C. Basso, T. Poggio, and T. Vetter. Reanimating Faces in Images and Video. In Eurographics, Granada, Spain, September 2003.

[5] M.E. Brand and A. Hertzmann. Style Machines. Computer Graphics, SIGGRAPH Proceedings, pages 183-192, July 2000.

[6] K. Choo and D.J. Fleet. People tracking using hybrid monte carlo fi ltering. In International Conference on Computer Vision, Vancouver, Canada, July 2001.

[7] CMU database. http://mocap.cs.cmu.edu/.

[8] A. J. Davison, J. Deutscher, and I. D. Reid. Markerless motion capture of complex full-body movement for character animation. In Eurographics Workshop on Computer Animation and Simulation. Springer-Verlag LNCS, 2001.

[9] J. Deutscher, A. Blake, and I. Reid. Articulated Body Motion Capture by Annealed Particle Filtering. In Conference on Computer Vision and Pattern Recognition, pages 2126-2133, Hilton Head Island, SC, 2000.

[10] A. Elgammal and C.S. Lee. Inferring 3D Body Pose from Silhouettes using Activity Manifold Learning. In CVPR, Washington, DC, June 2004.

[11] M. Isard. and A. Blake. CONDENSATION - conditional density propagation for visual tracking. International Journal of Computer Vision, 29(1):5-28, August 1998.

[12] A.D. Jepson, D. J. Fleet, and T. El-Maraghi. Robust on-line appearance models for vision tracking. IEEE Transactions on Pattern Analysis and Machine Intelligence, 25(10):1296-1311, 2003.

[13] V. Lepetit, A. Shahrokni, and P. Fua. Robust Data Association For Online Applications. In Conference on Computer Vision and Pattern Recognition, Madison, WI, June 2003.

[14] T.B. Moeslund. Computer Vision-Based Motion Capture of Body Language. PhD thesis, Aalborg University, Aalborg, Denmark, June 2003.

[15] T.B. Moeslund and E. Granum. A Survey of Computer Vision-Based Human Motion Capture. Computer Vision and Image Understanding, 81(3), March 2001.

[16] G. Mori, X. Ren, A.A. Efros, and J. Malik. Recovering Human Body Confi gurations: Combining Segmentation and Recognition. In Conference on Computer Vision and Pattern Recognition, Washington, DC, 2004. 
[17] D. Ormoneit, H. Sidenbladh, M.J. Black, and T. Hastie. Learning and tracking cyclic human motion. In Advances in Neural Information Processing Systems 13, pages 894-900, 2001.

[18] W.H. Press, B.P. Flannery, S.A. Teukolsky, and W.T. Vetterling. Numerical Recipes, the Art of Scientific Computing. Cambridge U. Press, Cambridge, MA, 1992.

[19] H. Sidenbladh, M. J. Black, and L. Sigal. Implicit Probabilistic Models of Human Motion for Synthesis and Tracking. In European Conference on Computer Vision, Copenhagen, Denmark, May 2002.

[20] C. Sminchisescu and B. Triggs. Kinematic Jump Processes for Monocular 3D Human Tracking. In Conference on Computer Vision and Pattern Recognition, volume I, Madison, WI, June 2003.

[21] J. Sullivan and S. Carlsson. Recognizing and tracking human action. In European Conference on Computer Vision, 2002.

[22] R. Urtasun and P. Fua. 3D Human Body Tracking using Deterministic Temporal Motion Models. In European Conference on Computer Vision, Prague, Czech Republic, May 2004.

[23] R. Urtasun, P. Glardon, R. Boulic, D. Thalmann, and P. Fua. Style-based Motion Synthesis. In Computer Graphics Forum. Eurographics Ass., To appear in December 2004. 